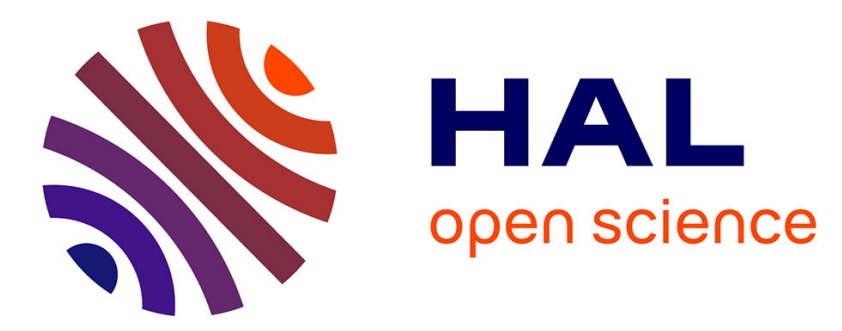

\title{
Visualisation et sortie de spectres enregistrés dans un IBM 1800
}

\author{
A. Muser, J. Zen, J.D. Michaud
}

\section{To cite this version:}

A. Muser, J. Zen, J.D. Michaud. Visualisation et sortie de spectres enregistrés dans un IBM 1800. Revue de Physique Appliquée, 1969, 4 (2), pp.145-146. 10.1051/rphysap:0196900402014500 . jpa00243187

\section{HAL Id: jpa-00243187 https://hal.science/jpa-00243187}

Submitted on 1 Jan 1969

HAL is a multi-disciplinary open access archive for the deposit and dissemination of scientific research documents, whether they are published or not. The documents may come from teaching and research institutions in France or abroad, or from public or private research centers.
L'archive ouverte pluridisciplinaire HAL, est destinée au dépôt et à la diffusion de documents scientifiques de niveau recherche, publiés ou non, émanant des établissements d'enseignement et de recherche français ou étrangers, des laboratoires publics ou privés. 


\title{
VISUALISATION ET SORTIE DE SPEGTRES ENREGISTRÉS DANS UN IBM 1800
}

\author{
A. MUSER, J. ZEN et J. D. MICHAUD, \\ Institut de Recherches Nucléaires, Strasbourg.
}

\begin{abstract}
Résumé. - La visualisation " programmée " utilise trop de temps pour renouveler les tables de sortie lorsque les données à visualiser évoluent rapidement. Nous avons réalisé une visualisation "semi-câblée " permettant l'acquisition, le traitement et la visualisation simultanément, et enfin une visualisation "câblée " pour augmenter la place mémoire réservée au traitement.

Abstract. - The "software visualisation" needs too much time to rewrite the data tables in the acquisition mode. We have developed a "hardware-software" visualisation system which permits simultaneously data acquisition, processing and display. A "hardware visualisation" increasing the size of the area available for the data processing is described.
\end{abstract}

Résumons les caractéristiques de l'ordinateur IBM 1800 intéressant le problème de la visualisation : 1) capacité de la mémoire : 32 kmots de 16 bits; 2) cycle de mémoire : $2 \mu \mathrm{s} ; 3$ ) registres d'entrée-sortie et de lecture par accès direct à la mémoire pouvant fonctionner en mode « canal », c'est-à-dire indépendamment du programme en cours.

I. Visualisation « programmée ». - On utilise un oscilloscope. Les seuls circuits nécessaires sont des convertisseurs digitaux-analogiques reliés aux entrées de l'oscilloscope. Dans le cas d'un spectre monoparamétrique, l'ordinateur doit fournir l'adresse $\mathrm{X}$ et le contenu C. Ceci ne présente aucune difficulté. En représentation biparamétrique, l'ordinateur doit fournir les coordonnées de l'image à partir des adresses $\mathrm{X}$ et $\mathrm{Y}$ et du contenu C. En ne transmettant qu'un mot par point, on peut visualiser $64 \times 64$ canaux en représentation isométrique avec possibilité de surbrillance et une définition de 7 bits sur le contenu. Dans ces conditions, le canal occupe $26 \%$ du temps machine avec une fréquence de répétition de l'image de 16/s.

Si l'on voulait visualiser 16384 canaux, il faudrait transmettre deux mots par point, le canal occuperait alors $100 \%$ du temps machine. Or ceci est inacceptable puisqu'au temps utilisé par le canal il faut ajouter le temps nécessaire pour préparer et renouveler les tables lorsque le spectre visualisé est en cours de formation (acquisition) : calcul des adresses $\mathrm{X}$ et $\mathrm{Y}$, transformation en isométrique, choix des bits significatifs pour le contenu, surbrillance.

II. Visualisation « semi-câblée ». - Si la visualisation doit fonctionner en simultanéité avec l'acquisition de données et un programme de traitement, il faut réduire au minimum le temps machine utilisé par la visualisation. Les mots constituant la table de sortie sont simplement transférés dans un certain ordre à partir de la zone de stockage, ce système de visualisation ne nécessitant pas la transmission des adresses.

Ce système comporte un registre d'adresse remis à zéro par un bit du premier mot de chaque table (c.-à-d. : image) et incrémenté par un oscillateur qui synchronise également le canal de sortie du 1800. La table étant chaînée sur elle-même, les images se succèdent sans interruption. Les bits significatifs sont choisis par un commutateur parmi les 15 bits du « motcontenu » et envoyés sur un convertisseur $\mathrm{D} / \mathrm{A}$. Le registre d'adresse ainsi que la sortie du convertisseur sont reliés à une unité de visualisation RG 96 (Intertechnique). Le système fonctionne correctement à 10 images/s et occupe pour 4096 canaux $8 \%$ du temps. La transmission du contenu seulement permet de visualiser un nombre quelconque de canaux en n'utilisant qu'un seul mot par canal (une modification du RG 96 permettrait par exemple de visualiser $16 \mathrm{kca}-$ naux en utilisant $32 \%$ du temps machine). Les paramètres déterminant le choix de la zone à visualiser sont choisis par le chercheur à l'aide de commutateurs (on peut choisir n'importe quelle zone telle que $\mathrm{XY}=4096)$. Un programme particulier permet de visualiser un spectre de 16384 canaux en le ramenant par intégration à 4096 canaux, l'intégration pouvant se faire sur X, sur Y ou sur X et Y. Ce système utilise peu de temps machine et peut fonctionner en simultanéité non seulement avec l'acquisition de données mais avec un programme de traitement ou d'entréesortie. La visualisation indique à ces programmes la zone à traiter. Pour désigner un point unique, on se sert du réticule du RG 96 qui provoque une interruption de programme au moment du passage du point. 
Les adresses $\mathrm{X}$ et $\mathrm{Y}$ de ce point sont affichées sur le RG 96, le contenu de ce point est affiché sur des tubes indicateurs numériques. Tous les appels de programmes se font à partir d'Ester à l'aide de touches et de boutonspoussoirs.

III. Visualisation « câblée ». — La simultanéité du fonctionnement de l'acquisition et de la visualisation avec le déroulement d'un programme de traitement limite la dimension de ce programme à 6 kmots environ. En effet, le système d'exploitation IBM occupe $4 \mathrm{kmots}$, la zone de stockage $16 \mathrm{kmots}$, le programme de visualisation 6 kmots.

La plupart des programmes de traitement étant écrits en langage Fortran, nous avons été amenés, pour gagner de la place en mémoire centrale, à considérer le programme de visualisation comme un programme de traitement parmi d'autres, ce programme n'étant appelé du disque que pour son exécution et ne permettant pas en général la simultanéité. Le programme de visualisation peut néanmoins être appelé par un programme d'exécution.

$\mathrm{Si}$ on veut visualiser un spectre en cours d'acquisition, on appelle le programme de visualisation de la zone de stockage avec renouvellement permanent de la table de sortie. Dans ce cas, aucune autre exé- cution de programme n'est possible simultanément.

Pour se libérer de cette contrainte et redonner la possibilité de contrôler le stockage par visualisation pendant l'exécution d'un programme, nous avons conçu une visualisation câblée sans programme enregistré. Trois modes de fonctionnement sont possibles :

III.1. L'adresse issue des codeurs est enregistrée dans une mémoire tampon qui est lue d'une part par le 1800 et d'autre part par le RG 96 (visualisation). Si le taux de comptage est suffisant, on obtient une représentation en projection.

III .2. Chaque fois que le 1800 a lu une adresse dans la mémoire tampon, il incrémente le mot correspondant (acquisition). Lorsqu'il signale qu'il a terminé, la visualisation lui demande le contenu du mot incrémenté. Ce contenu après conversion $\mathrm{D} / \mathrm{A}$ est transmis au RG 96 avec l'adresse lue sur la mémoire tampon.

III.3. Le fonctionnement est semblable à celui décrit en III .2, mais l'adresse est fournie par un registre incrémenté à partir d'un oscillateur. Le canal du 1800 fonctionne en acquisition et visualisation, mais les deux fonctions sont indépendantes. Les circuits doivent évidemment être conçus de façon à donner la priorité à l'acquisition. 